\title{
Evaluation of the effectiveness of ophthalmic assistants as screeners for glaucoma in North India
}

${ }^{1}$ Department of Cataract and Glaucoma Services, Venu Eye Institute and Research Centre, Sheikh Sarai Industrial Area, New Delhi, India

${ }^{2}$ International Centre for Eye Health, London School of Hygiene and Tropical Medicine, London, UK

Correspondence: SK Sinha, Venu Eye Institute and Research Centre, 1/31, Sheikh Sarai Institutional Area, Phase-2, New Delhi-110017, India Tel: + 919810789986 , + $911129251155 / 56$ +91 11 29250757;

Fax: + 9129252370.

E-mail: drsubodhsinha@ gmail.com

Received: 4 November 2010 Accepted in revised form: 17 May 2011 Published online: 1 July 2011

\begin{abstract}
Aim To assess whether ophthalmic assistants are effective in screening people for glaucoma in India.

Methodology The study subjects were examined by both trained ophthalmic assistants and an ophthalmologist in both hospital and community settings. Specific tests for the diagnosis of glaucoma suspects included visual field examination using frequency doubling technology perimetry, intraocular pressure measurement (Tonopen), A-scan central anterior chamber depth measurement and dilated optic disc examination. The findings recorded by the ophthalmic assistants were masked to the ophthalmologist to avoid measurement bias. Results In the hospital setting, there was a substantial level of agreement between the ophthalmic assistants and the ophthalmologist in the diagnosis of glaucoma suspects $(89.29 \%, k=0.7,95 \%$ confidence interval $(\mathrm{CI})=0.54-0.86)$. The diagnostic accuracy of the ophthalmic assistants in detecting glaucoma suspects was high for sensitivity (95.2\%, 95\% CI $=91.4-97.7 \%)$ but lower for specificity at $71.4 \%(95 \% \mathrm{CI}=60.0-78.7 \%)$. In the community setting, there was a moderate level of agreement between the ophthalmic assistants and the ophthalmologist

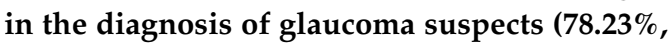
$k=0.50,95 \% \mathrm{CI}=0.37-0.64)$. The diagnostic accuracy of the ophthalmic assistants in detecting glaucoma suspects was moderate for sensitivity (82.9, 95\% CI=69.7-91.5\%) but lower for specificity at $76.8 \%(95 \% \mathrm{CI}=\mathbf{7 2 . 7 - 7 9 . 5 \% )}$. Conclusion Ophthalmic assistants can be used for opportunistic case detection of
\end{abstract}

SK Sinha ${ }^{1}$ and N Astbury ${ }^{2}$

glaucoma suspects in the community. Structured training of the ophthalmic assistants together with enhanced clinical experience would improve their performance in detecting glaucoma suspects in the community.

Eye (2011) 25, 1310-1316; doi:10.1038/eye.2011.154; published online 1 July 2011

Keywords: ophthalmic assistants; screeners; glaucoma

Introduction

Glaucoma is the second largest cause of blindness, among visual disorders, worldwide and also the leading cause of irreversible blindness. The number of people suffering from blindness globally due to glaucoma is estimated to be 4.5 million, accounting for approximately $12 \%$ of blindness. ${ }^{1}$ More importantly, most glaucoma patients remain asymptomatic until the disease is very advanced, with late presentation leading to irreversible blindness. ${ }^{2}$

In India, glaucoma is responsible for approximately $5.8 \%$ of blindness. ${ }^{3}$ A number of population-based cross-sectional studies on glaucoma in various parts of India show a prevalence of $2.6-4.39 \%$ in populations of 40 years and older. ${ }^{4-11}$ Importantly, of those identified with primary open-angle glaucoma (POAG) in these studies, more than $90 \%$ of subjects were diagnosed for the first time to have POAG and approximately one-fifth of those with POAG were blind due to glaucoma in one or both eyes. $5,7,12$ 
Results from various randomised-controlled trials ${ }^{13-17}$ support the view that the loss of visual function in most patients with ocular hypertension and glaucoma can be delayed by achieving a lower level of intraocular pressure (IOP). These studies stress the need for early detection and treatment of glaucoma patients to prevent irreversible loss of vision. The visual impairment and blindness caused by glaucoma imposes a huge socioeconomic burden both on the individual and society. Therefore, there is a need to develop methods and sufficient human resources for early detection of glaucoma in the community. Ophthalmic assistants can have a significant role in this regard.

Most of the studies ${ }^{18-23}$ on the effectiveness of ophthalmic paramedics have been carried out in developed countries in which the skill of the optometrists and environment in which they work is very different from that found in India. Very little information ${ }^{24}$ is available on the effectiveness of non-ophthalmologists especially ophthalmic assistants in glaucoma detection in the community in developing countries, especially India. 'Ophthalmic assistants' are ophthalmic paramedics trained formally for a period of 2 years. They are trained in refraction, diagnosis of eye diseases, community screening for major eye diseases and assisting ophthalmologists in hospitals in various aspects of eye care.

This study aims to establish whether ophthalmic assistants are effective in screening people for glaucoma in community setting in North India.

\section{Materials and methods}

The study was carried out during June/July 2009 in two phases - the initial 2 weeks in the hospital setting and the latter 2 weeks in the community setting. The study in the hospital setting was carried out in the glaucoma clinics of Venu Eye Institute and Research Centre, Delhi, India and its satellite hospital at Dhankot village, Gurgaon in the province of Haryana. The study in the community setting was carried out during the glaucoma screening camps organised in the Mewat district of Haryana province in North India.

\section{Study design}

This was a directly comparative, masked, performance study involving individuals visiting glaucoma clinics or high-risk individuals visiting screening camps. The clinical findings and diagnoses of the ophthalmic assistants were masked to the ophthalmologist. In the hospital setting, to avoid any measurement bias, both ophthalmic assistants and ophthalmologist were also masked to the hospital records of the patients.

\section{Sample size calculation}

The sample size was calculated using Epi-info version 6.04 (Centre for Disease Control and Prevention, Atlanta, GA, USA). The sample size calculation was based on an estimated $10 \%$ disagreement in diagnosis between ophthalmic assistants and ophthalmologists with a 95\% confidence interval (CI) of the width 5\% (ie, 5-15\%). For this, at least 138 hospital subjects and 138 subjects in the community (a total of 276 subjects) needed to be examined by both ophthalmic assistants and ophthalmologist.

The definition of 'glaucoma suspect' in this study was based on the International Society of Geographical and Epidemiologic Ophthalmology classification proposed by Foster et al..$^{25}$ One or more of the following criteria were used for the diagnosis of a glaucoma suspect:

1. Optic disc cupping - eyes with a cup disc ratio or cup disc ratio asymmetry $>97.5$ th percentile for the normal population, that is, vertical cup disc ratio (VCDR) $\geq 0.7$ or asymmetry of the cup disc ratio of $\geq 0.2$ between two eyes with symmetrical disc diameters.

2. Optic disc margin haemorrhages.

3. Visual field abnormalities - considered abnormal if one point was abnormal on frequency doubling technology (FDT) perimetry (C 24-2-1 programme).

4. $\mathrm{IOP} \longrightarrow>97.5$ th percentile, that is, $>21 \mathrm{~mm} \mathrm{Hg}$.

5. Occludable drainage angle - central anterior chamber depth $(A C D)<2.53 \mathrm{~mm}$.

The 97.5 percentile for IOP and VCDR for the Indian population was defined based on a population based study carried out in South India. ${ }^{4}$ Measurement of the central ACD for the detection of occludable angles was based on a study carried out in an East Asian population. ${ }^{26}$ Owing to the unavailability of pachymeter, central ACD using A-scan was used as a surrogate for true ACD.

Four ophthalmic assistants from the Venu Eye Institute and Research Centre were invited to participate in the training. All of them had more than 2 years of working experience, including outreach eye care activities. The training of the ophthalmic assistants included 3 days of structured training together with a $1 \mathrm{~h}$ feedback session each week during the study. The same ophthalmic assistants were used for the study of both groups of study subjects.

For the hospital settings the study subjects were selected from patients visiting glaucoma clinic for screening or follow up. For the community setting the study subjects were selected from special camps for glaucoma screening. The subjects not willing to participate in the study were excluded. 
Each study subject was examined by the ophthalmic assistants and ophthalmologist separately. The examination included recording the presenting vision and best-corrected vision (using an E-type Snellen chart) and anterior segment examination using torchlight. Specific tests for the diagnosis of glaucoma suspects included visual field examination using FDT perimetry (Humphrey Matrix Perimeter, Carl Zeiss Meditec Inc., Dublin, CA, USA, using 24-2-1 screening programme), IOP measurement using a Tonopen (Reichert Tono-Pen XL Tonometer, Reichert Inc., Depew, NY, USA), central ACD measurement using an A-scan (Ocuscan, Alcon Laboratories Inc., Fort Worth, TX, USA) and dilated optic disc examination using direct ophthalmoscopy (Heine Beta 200 Ophthalmoscope, Heine Optotechnik GmbH \& Co., Herrsching, Germany).

The study was conducted in accordance with the principles of the Declaration of Helsinki and had the approval of the ethics committees of the London School of Hygiene and Tropical Medicine, as well as the Venu Eye Institute and Research Centre, Delhi. Written informed consent from the all study subjects was taken by the ophthalmic assistants before the inclusion in the study.

Data analysis was performed by the researcher using STATA v.10 (Statacorp, College Station, TX, USA) and $\kappa$-statistics were used to assess agreement on nominal variables such as the diagnosis of glaucoma suspects and FDP results. Weighted $\kappa$-statistics were used to assess agreement between ophthalmic assistants and the ophthalmologist on ordinal variables, such as vertical cup disc ratio, keeping in mind the importance of different degrees of disagreement. Agreement on continuous variables such as IOP measurements and ACD were assessed using intraclass correlation coefficient.

\section{Results}

\section{Study population}

A total of 140 subjects in the hospital setting and 147 subjects in the community setting were enrolled for the study. In all, 278 eyes in the hospital and 291 eyes in the community setting were examined by both ophthalmic assistants and an ophthalmologist. The demographic variables, personal history and family history of study subjects are summarised in Table 1.

The difference in the mean age $(P=0.43)$ and gender $(P=0.21)$ in the community and hospital settings was not statistically significant, although only subjects aged 40 years or more were included in the camp setting. A significant proportion of subjects $(37.8 \%)$ were known cases of glaucoma in the hospital setting compared with the study subjects in the community setting $(4.76 \%$ previously diagnosed glaucoma) as they were enrolled from the glaucoma clinics.

\section{Interobserver agreement between the ophthalmic assistants}

After 3 days of training and before the start of the study, the interobserver agreement on 50 eyes of 25 patients revealed good agreement between the ophthalmic assistants for the diagnosis of glaucoma suspects $(k=0.70,95 \% \mathrm{CI}=0.43-0.97)$.

\section{Agreement on diagnosis of glaucoma suspects}

To assess the agreement between the ophthalmic assistants and ophthalmologist for the diagnosis and individual test results, results from the two ophthalmic assistants were pooled and compared with those of the ophthalmologist. A diagnosis of 'glaucoma suspect' was made in 105 subjects by the ophthalmologist and in 110 subjects by the ophthalmic assistants in the hospital setting. In the community setting, a diagnosis of 'glaucoma suspect' was made in 35 subjects by the ophthalmologist and in 55 subjects by the ophthalmic assistants.

There was good agreement between the ophthalmic assistants and the ophthalmologist in the diagnosis of glaucoma suspects in the hospital setting $(89.29 \%, k=0.7,95 \% \mathrm{CI}=0.54-0.86)$ but the level of agreement was moderate in the community setting $(78.23 \%, k=0.50,95 \% \mathrm{CI}=0.37-0.64)$. The sensitivity, specificity and positive predictive value of ophthalmic assistants in diagnosis of glaucoma suspects in hospital and community settings are shown in Table 2.

Table 1 Demographic variables, history (personal and family history) of study subjects

\begin{tabular}{lcccccr}
\hline Setting & $\begin{array}{c}\text { Number of study } \\
\text { subjects }\end{array}$ & $\begin{array}{c}\text { Age (years) } \\
\text { mean } \pm \text { s.d. }\end{array}$ & Male & Female & $\begin{array}{c}\text { Previously } \\
\text { diagnosed glaucoma }\end{array}$ & $\begin{array}{c}\text { Family history } \\
\text { of glaucoma }\end{array}$ \\
\hline Hospital & 140 & $53.13 \pm 15.27$ & 77 & 63 & 53 & 3 \\
Community & 147 & $54.36 \pm 11.12$ & 70 & 77 & 7 & 2 \\
\hline
\end{tabular}


Table 2 Sensitivity/specificity and positive predictive value of ophthalmic assistants in diagnosis of glaucoma suspects in hospital and community settings

\begin{tabular}{lcc}
\hline & Value & $\begin{array}{c}95 \% \text { confidence } \\
\text { interval }\end{array}$ \\
\hline $\begin{array}{l}\text { Hospital setting } \\
\quad \text { Sensitivity }\end{array}$ & 95.2 & $91.4-97.7$ \\
$\quad$ Specificity & 71.4 & $60-78.7$ \\
$\quad$ Positive predictive value & 90.9 & $87.3-93.2$ \\
& & \\
Community setting & & $69.7-91.5$ \\
$\quad$ Sensitivity & 82.9 & $72.7-79.5$ \\
$\quad$ Specificity & 76.8 & $44.3-58.2$ \\
\hline Positive predictive value & 52.7 & \\
\hline
\end{tabular}

Table 3 Level of agreement in different tests between ophthalmic assistants and ophthalmologist in hospital and community settings using $\kappa$-statistic

\begin{tabular}{|c|c|c|c|c|}
\hline \multirow{2}{*}{$\begin{array}{l}\text { Test variable } \\
\text { Hospital settin }\end{array}$} & \multirow[t]{2}{*}{ Agreement } & \multirow[t]{2}{*}{$\kappa$} & \multicolumn{2}{|c|}{$\begin{array}{c}\kappa 95 \% \text { confidence } \\
\text { interval }\end{array}$} \\
\hline & & & & \\
\hline FDP & 93.91 & 0.91 & 0.83 & 0.99 \\
\hline VCDR & 91.19 & 0.74 & 0.66 & 0.82 \\
\hline \multicolumn{5}{|c|}{ Community setting } \\
\hline FDP & 90.38 & 0.83 & 0.75 & 0.91 \\
\hline VCDR & 92.86 & 0.52 & 0.45 & 0.59 \\
\hline
\end{tabular}

\section{Agreement on individual test results}

In the hospital setting, the agreement between the ophthalmic assistants and ophthalmologist for the assessment of visual fields using FDT perimetry was very good (agreement 93.9\%, $\kappa=0.91$ ), whereas there was good agreement for the assessment of VCDR (agreement $91.2 \%, K_{\mathrm{w}}=0.74$ ). In the community setting, the agreement between the ophthalmic assistants and ophthalmologist for the assessment of visual fields using FDT perimetry was very good (agreement $90.38 \%$, $\kappa=0.83$ ), whereas there was moderate agreement for the assessment of VCDR (agreement 92.68\%, $K_{w}=0.52$; Table 3).

In the hospital setting, calculation of the intraclass correlation coefficient indicates that there was good agreement between the ophthalmic assistants and ophthalmologist for the measurement of IOP (ICC 0.84, $95 \% \mathrm{CI}=0.81-0.88)$ and substantial agreement in assessment of ACD (ICC $0.78,95 \% \mathrm{CI}=0.74-0.83$ ). In the community setting, calculation of the intraclass correlation coefficient indicates that there was very good agreement between the ophthalmic assistants and ophthalmologist for the measurement of IOP (ICC 0.95, $95 \% \mathrm{CI}=0.94-0.96)$ and substantial agreement for
Table 4 Agreement in different tests between ophthalmic assistants and ophthalmologist in hospital and community settings using intraclass correlation

\begin{tabular}{lccc}
\hline Test variables & $\begin{array}{c}\text { Intraclass } \\
\text { correlation }\end{array}$ & $\begin{array}{c}\text { Standard } \\
\text { error }\end{array}$ & $\begin{array}{c}95 \% \text { confidence } \\
\text { interval }\end{array}$ \\
\hline $\begin{array}{l}\text { Hospital setting } \\
\quad\end{array}$ & & & \\
$\quad$ IOP & 0.84 & 0.0173 & $0.81-0.88$ \\
$\quad$ Anterior chamber depth & 0.78 & 0.0233 & $0.74-0.83$ \\
& & & \\
Community setting & & & \\
$\quad$ IOP & 0.95 & 0.00598 & $0.94-0.96$ \\
$\quad$ Anterior chamber depth & 0.71 & 0.0233 & $0.65-0.77$ \\
\hline
\end{tabular}

assessment of ACD (ICC 0.71, 95\% CI =0.65-0.77;

Table 4).

\section{Discussion}

This study was carried out to assess the effectiveness of ophthalmic assistants in screening people for glaucoma in both hospital and community settings in India. The hospital setting was used to increase the number of glaucoma/glaucoma suspect patients in the study. This in turn led to greater validity in assessing the effectiveness of ophthalmic assistants in diagnosing glaucoma suspects. The ophthalmic assistants taking part in this study had very limited previous experience in working with the protocols and equipment used in this study. However, there was good interobserver agreement among the ophthalmic assistants after their training and before the commencement of the study. These factors increase the external validity of the study for the ophthalmic assistants working in other eye care organisations in India.

The relatively higher than expected proportion of subjects diagnosed as glaucoma suspects in the community setting (23.8\%), based on population based prevalence studies in India, ${ }^{4-10}$ could be explained by different criteria used for the diagnosis of glaucoma suspects compared with those used to diagnose glaucoma. The patients visiting the screening camps cannot be considered to be representative of the population. This is supported by the fact that 7 out of 35 subjects $(20 \%)$ diagnosed by the ophthalmologist as glaucoma suspects in the screening camps were known glaucoma patients and were on treatment. Furthermore, three patients were diagnosed as glaucoma suspects solely on the basis of ACD.

The glaucoma awareness campaign undertaken by the field workers could also have resulted in an increased number of glaucoma suspect patients visiting the screening camps. This suggests a possible role for field 
workers in increasing glaucoma awareness in the community.

Masking of the ophthalmologist and the ophthalmic assistants to the clinical records in the hospital setting was carried out to avoid any bias in the diagnosis of glaucoma suspects. Good agreement between ophthalmic assistants and ophthalmologists in the hospital setting in the diagnosis of glaucoma $(88.21 \%$, $k=0.74$, s.e. $=0.0582$ ) suggests that ophthalmic assistants can readily detect glaucoma suspect patients at an advanced stage and in a supportive environment. A moderate level of agreement $(78.23 \%, k=0.50$, s.e. $=$ 0.078 ) in the community setting suggests that more extensive training will be required for detection of early cases of glaucoma by the ophthalmic assistants. The level of agreement in the hospital setting is similar to the agreement found between accredited glaucoma optometrists and consultant ophthalmologists in the diagnosis of glaucoma in the hospital setting in a study done by Azura Blanco et al. ${ }^{27}$

The high sensitivity in diagnosing glaucoma suspects $(95.2,95 \% \mathrm{CI}=91.4-97.7 \%)$ in the hospital setting can be explained by study subjects being at an more advanced stage of glaucoma, use of multiple clinical tests to identify glaucoma suspects and a better environment for conducting tests (availability of dark room, adequate time). The moderate sensitivity in the community setting $(82.9,95 \% \mathrm{CI}=69.7-91.5 \%)$ can be explained by the presence of early glaucoma suspect patients and fewer patients completing reliable FDT perimetry in the community setting. The low specificity in the hospital setting could be explained by the learning curve of the ophthalmic assistants for various new clinical tests as well as ophthalmic assistants being overcautious. Better specificity (moderate) in the community setting compared with the hospital setting could be explained by the ophthalmic assistants benefitting from their hospital experience, including use of equipment, before undertaking the study in the community. This emphasises the need for continuous training and regular feedback for any ophthalmic paramedic involved in glaucoma screening. The low positive predictive value (PPV; 52.7, 95\% CI $=44.3-58.3 \%$ ) of the glaucoma suspects in the community setting is similar to the study by Bowling et $\mathrm{al}^{28}$ in which nearly half $(45.8 \%)$ of the patients referred from community primary care sources were discharged from ophthalmological review. The low PPV can result in a large number of unnecessary referrals from the ophthalmic assistants to the hospital services.

Of the clinical test measurements studied, the highest agreement between ophthalmic assistants and ophthalmologist was observed with visual field examination using FDT perimetry ( $k$-value of 0.91 and 0.83 in hospital and community settings, respectively).
A similar outcome was observed in a study conducted by Spry et $a l^{29}$ comparing measurement of cup to disc ratio, visual field score and IOP taken by community optometrists with research clinic reference standards. Visual field assessment was found to be the most reliable measurement and the cup to disc ratio the least reliable in this study. There was much better agreement on IOP measurement in the community setting compared with the hospital setting. The ophthalmic assistants were more used to measuring IOP with a Tonopen by the time the study started in the community setting. The hospital setting had more patients with high IOP ranges and measurement of IOP with a Tonopen is known to be less accurate at extreme values. ${ }^{30,31}$ There was moderate level of agreement $(k=0.52$, s.e. $=0.0359)$ on VCDR

assessment in the community setting. Nevertheless the precision was good enough $(k=68.25$, s.e. $=0.06)$ for the purpose of adequate screening, that is, to detect whether the optic disc is glaucomatous or not (VCDR $\geq$ or $<0.7)$.

This study had several limitations. Reliable results from FDT perimetry for the assessment of visual fields were obtained from approximately $45 \%$ of the eyes in the community setting and $75 \%$ of the eyes in the hospital setting. Unreliable test results (high fixation errors, falsepositive results), low vision and instrument malfunction in one screening camp were all to blame. Unreliable test results in the community setting can be attributed to an unsupportive environment. This could have lead to under diagnosis of glaucoma suspects. Although previous studies have confirmed the potential of central ACD measurement as a screening tool for angle closure in an East-Asian population, ${ }^{26}$ the surrogate use of central ACD using A-scan for true SCD for occludable angles and its specificity/sensitivity in detecting occludable angles in an Indian population requires further study.

Enhanced training of ophthalmic assistants could have resulted in better agreement between ophthalmic assistants and the ophthalmologist. Increasing the number of ophthalmic assistants and ophthalmologists could have resulted in more external validity in the study. Although clinical records were masked to the ophthalmic assistants, a positive history of glaucoma and use of glaucoma medications could have caused some bias in the diagnosis of glaucoma suspects in the hospital setting.

The study indicates a substantial and encouraging level of agreement between ophthalmic assistants and an ophthalmologist in the diagnosis of glaucoma in the hospital setting and a moderate level of agreement in the community setting. More extensive clinical experience would improve the diagnostic sensitivity and specificity of the ophthalmic assistants enabling them to more reliably detect glaucoma suspects in the community. 


\section{Summary}

\section{What was known before}

- Ongoing training of optometrists in western settings resulted in an increased rate of detection of glaucoma in the community.

- Many studies, in western settings, have been carried out to determine the effectiveness of optometrists in glaucoma detection, referral and management decisions. These studies project a mixed picture concerning the effectiveness of optometrists in the community detection of glaucoma as well as their decision to treat.

\section{What this study adds}

- Ophthalmic assistants can be used for opportunistic case detection of glaucoma suspects in the community in India.

- Structured training of the ophthalmic assistants including periodic refresher courses together with continuous feedback on the referred patients can help in increasing their effectiveness to identify glaucoma suspects.

\section{Conflict of interest}

The authors declare no conflict of interest.

\section{Acknowledgements}

We thank the Commonwealth Scholarship Commission, the Department for International Development for the financial support, all ophthalmic assistants, communitybased rehabilitation workers and the study participants for taking part in this study.

\section{References}

1 Resnikoff S, Pascolini D, Etya'ale D, Kocur I, Pararajasegaram R, Pokharel GP et al. Global data on visual impairment in the year 2002. Bull World Health Organ 2004; 82(11): 844-851.

2 Hitchings RA. Glaucoma screening. Br J Ophthalmol 1993; 77(6): 326.

3 Murthy GV, Gupta SK, Bachani D, Jose R, John N. Current estimates of blindness in India. Br J Ophthalmol 2005; 89(3): 257-260.

4 Vijaya L, George R, Baskaran M, Arvind H, Raju P, Ramesh SV et al. Prevalence of primary open-angle glaucoma in an urban south Indian population and comparison with a rural population. The Chennai Glaucoma Study. Ophthalmology 2008; 115(4): 648-654.

5 Vijaya L, George R, Arvind H, Baskaran M, Ve Ramesh S, Raju $\mathrm{P}$ et al. Prevalence of primary angle-closure disease in an urban south Indian population and comparison with a rural population. The Chennai Glaucoma Study. Ophthalmology 2008; 115(4): 655-660.

6 Ramakrishnan R, Nirmalan PK, Krishnadas R, Thulasiraj RD, Tielsch JM, Katz J et al. Glaucoma in a rural population of southern India: the Aravind comprehensive eye survey. Ophthalmology 2003; 110(8): 1484-1490.

7 Dandona L, Dandona R, Mandal P, Srinivas M, John RK, McCarty CA et al. Angle-closure glaucoma in an urban population in southern India. The Andhra Pradesh eye disease study. Ophthalmology 2000; 107(9): 1710-1716.

8 Dandona L, Dandona R, Srinivas M, Mandal P, John RK, McCarty CA et al. Open-angle glaucoma in an urban population in southern India: the Andhra Pradesh eye disease study. Ophthalmology 2000; 107(9): 1702-1709.

9 Palimkar A, Khandekar R, Venkataraman V. Prevalence and distribution of glaucoma in central India (Glaucoma Survey 2001). Indian J Ophthalmol 2008; 56(1): 57-62.

10 Raychaudhuri A, Lahiri SK, Bandyopadhyay M, Foster PJ, Reeves BC, Johnson GJ. A population based survey of the prevalence and types of glaucoma in rural West Bengal: the West Bengal Glaucoma Study. Br J Ophthalmol 2005; 89(12): $1559-1564$.

11 Jacob A, Thomas R, Koshi S, Braganza A, Muliyil J. Prevalence of primary glaucoma in an urban south Indian population. Indian J Ophthalmol 1998; 46(2): 81-86.

12 George R, Vijaya L. First World Glaucoma day, March 6, 2008: tackling glaucoma challenges in India. Indian J Ophthalmol 2008; 56(2): 97-98.

13 Kass MA, Heuer DK, Higginbotham EJ, Johnson CA, Keltner JL, Miller JP et al. The Ocular Hypertension Treatment Study: a randomized trial. Arch Ophthalmol 2002; 120(6): 701-713; discussion 829-830.

14 The AGIS Investigators. The Advanced Glaucoma Intervention Study (AGIS): the relationship between control of intraocular pressure and visual field deterioration. Am J Ophthalmol 2000; 130(4): 429-440.

15 Leske MC, Heijl A, Hussein M, Bengtsson B, Hyman L, Komaroff E. Factors for glaucoma progression and the effect of treatment: the early manifest glaucoma trial. Arch Ophthalmol 2003; 121(1): 48-56.

16 Heijl A, Leske MC, Bengtsson B, Hyman L, Hussein M. Reduction of intraocular pressure and glaucoma progression: results from the Early Manifest Glaucoma Trial. Arch Ophthalmol 2002; 120(10): 1268-1279.

17 Collaborative Normal-Tension Glaucoma Study Group. Comparison of glaucomatous progression between untreated patients with normal-tension glaucoma and patients with therapeutically reduced intraocular pressures. Am J Ophthalmol 1998; 126(4): 487-497.

18 Johnson ZK, Griffiths PG, Birch MK. Nurse prescribing in glaucoma. Eye 2003; 17(1): 47-52.

19 Wormald RP, Rauf A. Glaucoma screening. J Med Screen 1995; 2(2): 109-114.

20 Banes M, Culham L, Crowston J, Bunce J, Khaw P. An optometrist's role of comanagement in a hospital glaucoma clinic. Ophthal Physiol Opt 2000; 20: 351-359.

21 Oster J, Culham LE, Daniel R. An extended role for the hospital optometrist. Ophthalmic Physiol Opt 1999; 19(4): 351-356.

22 Theodossiades J, Murdoch I, Cousens S. Glaucoma case finding: a cluster-randomised intervention trial. Eye 2004; 18(5): 483-490.

23 Henson DB, Spencer AF, Harper R, Cadman EJ Community refinement of glaucoma referrals. Eye 2003; 17(1): 21-26. 
24 Foster A, Wormald R, van de Heide A, Templeton K, Minassian D. Evaluation of ophthalmoscopy by nonophthalmologists in diagnosing chronic glaucoma in West Africa. Eye 1989; 3(5): 647-650.

25 Foster PJ, Buhrmann R, Quigley HA, Johnson GJ. The definition and classification of glaucoma in prevalence surveys. Br J Ophthalmol 2002; 86(2): 238-242.

26 Devereux JG, Foster PJ, Baasanhu J, Uranchimeg D, Lee PS, Erdenbeleig $\mathrm{T}$ et al. Anterior chamber depth measurement as a screening tool for primary angle-closure glaucoma in an East Asian population. Arch Ophthalmol 2000; 118(2): 257-263.

27 Azuara-Blanco A, Burr J, Thomas R, Maclennan G, McPherson S. The accuracy of accredited glaucoma optometrists in the diagnosis and treatment recommendation for glaucoma. Br J Ophthalmol 2007; 91(12): 1639-1643.
28 Bowling B, Chen SD, Salmon JF. Outcomes of referrals by community optometrists to a hospital glaucoma service. $\mathrm{Br} J$ Ophthalmol 2005; 89(9): 1102-1104.

29 Spry PG, Spencer IC, Sparrow JM, Peters TJ, Brookes ST, Gray $S$ et al. The Bristol Shared Care Glaucoma Study: reliability of community optometric and hospital eye service test measures. Br J Ophthalmol 1999; 83(6): 707-712.

30 Lester M, Mermoud A, Achache F, Roy S. New Tonopen XL: comparison with the Goldmann tonometer. Eye 2001; 15(Part 1): 52-58.

31 Bandyopadhyay M, Raychaudhuri A, Lahiri SK, Schwartz EC, Myatt M, Johnson GJ. Comparison of Goldmann applanation tonometry with the Tonopen for measuring intraocular pressure in a population-based glaucoma survey in rural West Bengal. Ophthalmic Epidemiol 2002; 9(3): 215-224. 